\title{
MANAGEMENT OF POST-BURN CONTRACTURES
}

\author{
Schwarz R J, Joshi K D*
}

\section{INTRODUCTION}

Post-burn contractures are distressingly common in Nepal and India as well as in other developing nations. Rolling of infants into unprotected fires or explosions of poorly made stoves are the most common causes of these burns. Failure to seek medical help, inadequate medical care and inadequate post-healing care are all common causes of burn contractures. All wounds which heal by secondary intention will heal by the processes of epithelisation and contraction. Contraction is a healing process that decreases the size of a wound by fibroblastic activity pulling the edges of a wound together. The pain from a burn wound will tend to make the patient place the joint in a position with the least tension on the wound (position of comfort). As the process of contraction proceeds, the joint or other structure (lip, eyelid) is pulled more and more towards the center of the wound, the end result being a contracture.

\section{PREVENTION}

Obviously, the best means of prevention is avoiding the burn in the first instance. A great deal needs to be done in the education of parents regarding fire safety and the construction of safe fire pits and stoves. However, once the burn has occurred, the best means of prevention of burn contractures is facilitating rapid primary healing of superficial burns or early skin grafting of deep burns. Split thickness grafts are usually used, although full thickness grafts to small burns on the hand and face are advantageous as they contract less, have a better cosmetic match and are more durable.

During the time of wound healing and continuing until the scar is mature, splinting the limb in the position of function is important. Night splinting is often adequate, and this process may need to continue for up to two years following the burn. ${ }^{1}$ Parental and patient compliance with this program is very difficult to achieve. Physiotherapy of the involved limb (both active and passive exercises) is likewise important to maintain a complete range of motion. Application of a moisturising oil or cream and massage of the scar is also important in producing a healthy scar with a lower likelihood of contracture. Prevention of burn contracture is definitely preferable to treatment of the same.

\section{PRINCIPLES OF MANAGEMENT}

Burn contractures, especially severe ones, have a tendency to recur, especially in a growing child. Surgery is directed at reducing this tendency. Before attempting release, the patient should be asked to wait preferably a year from the time of the burn, which can be frustrating to the patient. However during this time massage, splinting and regular visits to the physiotherapist will pay dividends in the end. The amount of release that can be obtained at one time is often limited by associated contractures of joints, tendons and ligaments. It is often better to carry out two simple procedures, such as skin grafts or local flaps, than more complex procedures such as distant flaps. The initial procedure will get the affected limb in a position in which further exercises and serial splinting can achieve a significant lengthening of joint structures, after which a second procedure can achieve complete release of the contracture. If tendons, bone or joints are exposed during the release of the contracture, the use of a flap is imperative. The use of flaps over joints will definitely decrease the recurrence rate due to inclusion of normal skin and subcutaneous tissue, and splinting need not be done more than about two weeks. ${ }^{2-4}$ Following a graft, the patient's compliance is needed to maintain splinting from 6 months to one year. Distant flaps,

* Green Pastures Hospital and Rehabilitation Center, Pokhara, Nepal.

** Bir Hospital, Kathmandu, Nepal.

Address for correspondence : Dr. Richard J Schwarz

Green Pastures Hospital and Rehabilitation Center

P.O. Box: 28, Pokhara, Nepal

Email: gph@inf.org.np 
especially in the hand, are bulky and give a less than optimal functional and cosmetic result. ${ }^{5}$ If the scar tissue is of very poor quality, or the scar is unstable (recurring ulcers) then excision of this part of the scar is advisable.

Often multiple limbs are involved. It is important for the surgeon to make an overall plan, and to carry out as many procedures at each operative session as is reasonable in order to minimize the number of aneasthetics. A well-planned surgical program will give cost-savings to the patient and as well will shorten the rehabilitation phase.

The completeness of release that can be expected from a procedure depends on the age of the patient, the age of the burn injury and the severity of the deformity.

a) The younger the patient the less one will expect joint stiffness to prevent the return of a full range of motion after a skin release. Usually up to the age of 12 one can expect the return of a complete range of motion.

b) The longer the deformity has been present the less likely a full range of motion will be obtained, at least initially. Tendons, ligaments and joint capsules will all have adapted to the deformity by shortening on the involved side. Bony deformity can be seen as the patient grows, such as mandibular curvature with neck contractures, and these can be very difficult to correct. The joint can develop in a dislocated position, making future normal function of the joint impossible.

c) The more severe the deformity, the more severe one can expect limitation of release by the shortening of associated structures, as well as by bony and joint maldevelopment as noted above.

The release should be carried down through all scar tissue to obtain optimal release Excise as much scar as reasonable to obtain maximum release of the joint. A scalpel is usually best. After cutting mid-axis to mid-axis over the joint then "push" with the knife as someone stretches the joint so that only what is scar will be cut. Constant tension is required to allow identification of areas of tightness. I find that a towel clip through the tip of a digit gives secure traction and avoids having the digit slip during the release. The release will generally need to go down to the midpoint of the joint to obtain complete release. ${ }^{1,6}$ One must be aware of important structures (nerves, vessels) in danger of being traumatized during the release. Another risk is over-zealous correction leading to vessel spasm and possible digit/ limb loss, although the authors have never personally experienced this problem.

Infection of the graft is disastrous. We give a single dose of antibiotic. More important is proper preparation of the patient including both the involved limb and the donor limb. There are often hidden pockets that can be difficult to clean, and these must be sought out and thoroughly cleaned.

Specific techniques used are as follows:

a) Release and skin grafting

The release is usually a fishmouth type of incision (Fig.1). ${ }^{1,6}$ although one finds that often one of the lateral

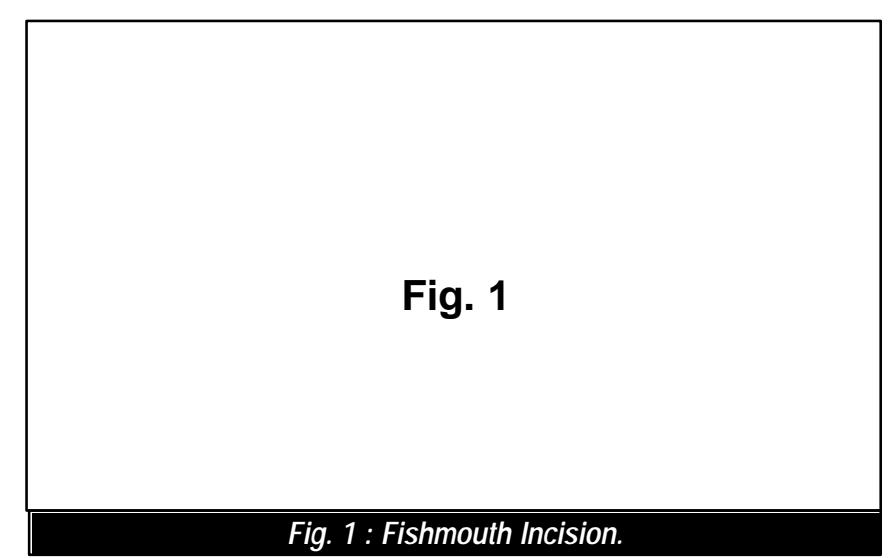

incisions does not open up and does not really contribute to the release. The remaining defect is surfaced with full thickness skin graft if at all possible. Split-thickness plantar skin grafts will give similarly excellent colour matching with minimal contraction. ${ }^{6,7,8}$ Both will give a more durable and cosmetically pleasing result than a split thickness graft, and will be much less likely to contract. ${ }^{6,9,10}$ Large full thickness grafts can be obtained from the groin or suprapubic areas with minimal donor defect. If the defect is too large then split thickness skin can be used, but this is definitely second choice. One can combine both techniques, using full thickness graft at the joint line and split thickness adjacent to this. A calculation of the size of the defect expected can be made by drawing a line from the points of the contracture across to the contralateral side of the limb and measuring the length between these two points. Pressure over the graft is important post-operatively, which is effectively obtained with tie-over dressings and/or circumferential bandaging.

b) Local Flaps

Where possible a flap is the best for joints, and perhaps the commonest is the $\mathrm{Z}$ plasty (see below). Other common flaps are Y-V, V-Y (see below) or trapezoidplasty, where the chance of corner necrosis is less. The flaps should include all subcutaneous fat. Half buried horizontal mattress sutures can be used in the corners to cause the least damage to the blood supply. The post-operative care is much easier, as therapy can be started early and the need for splinting is reduced. The cosmetic result is better as the darkening usually seen in skin grafting is avoided. They are preferable to distant flaps due to their simplicity and avoidance of maintaining the limb in an uncomfortable position. Other flaps that have been used 
are digital island flap, fillet flap, lateral or dorsolateral flap, dorsometacarpal flap, cross finger flap, flag flap, and the adipofascial turnover flap. . $^{1,6,11,12}$

\section{Z-plasty}

This flap is principally useful in the treatment of linear band contractures. It is at high risk of partial necrosis of part if both flaps are not comprised of mostly healthy normal tissue.1,13,14 The Z-plasty can be successfully used in combination with skin grafting to reduce the amount of skin graft used. Care must be taken in this instance as flap necrosis and infection can then lead to graft loss. With unhealthy tissue a partial transposition of the flaps can be used (Fig. 2) which has the advantage of staggering

Fig. 2

Fig. 2 : Partial Z-plasty with 90 degree angles.

the skin graft and thereby reducing the recurrent contracture risk. If only one side is scarred one can do a half $\mathrm{Z}$ plasty where on one side the angle can be about 90 degrees. Z-plasties can be single or multiple.

\section{V-Y plasty (Fig. 3)}

This is suitable for those burns with a narrow band of contracture with healthy skin on either side. ${ }^{13,14}$

\section{Fig. 3}

Undermining is neither necessary nor advisable due to the risk of skin necrosis. Usually multiple V-Y plasties are needed, and interposing healthy skin between burned segments can be very successful. It has a lower risk of flap necrosis than the Z-plasty. ${ }^{13,14}$ This technique will not work when there is a wide area of thickened burned skin across a joint.

\section{C) Regional Flaps}

Flaps such as the radial forearm fasciocutaneous flap, posterior interosseous artery flap, latisimus dorsi flap or the posterior calf flap are reliable with minimal donor morbidity.

D) Distant flaps

These are usually not required, and the tissue bulk and the time spent operatively or awaiting vascularization of the flap prior to division makes these flaps a method of last choice in most instances. Useful examples would be a groin flap, Louvre flap, abdominal flap, or cross arm flap for hand defects, or free flaps.,11,12

E) Ilizarov Technique

Gradual distraction of a contracted joint can also be used to restore a joint to its normal position..$^{15}$ This has the advantage of stretching the underlying soft tissues and joint capsule at the same time. Disadvantages include the prolonged time required in the devise, the risk of pin-tract infections, and increased expense.

F) Tissue expansion

Tissue expansion is often used in burns to obtain local skin with an excellent match. ${ }^{16}$

\section{SPECIFIC POINTS BY ANATOMIC LOCATION}

\section{Eyelid}

Burn scar above or below the eye with contracture will cause ectropion. This is a major cosmetic problem. The inability to close the lids causes the cornea to remain exposed, leaving the eye at risk of trauma and corneal drying. Moisturizing drops and teaching the patient to consciously close the eye intermittently help to control corneal drying until surgery can be carried out. Surgery consists of release of any contracture present and replacement with full thickness skin graft. Some authors use thick split-thickness skin for the upper lid. The release must extend beyond the medial and lateral canthus. ${ }^{17}$ Over-correction is recommended by some authors..$^{16}$ The best match is from either the clavicular or post-auricular areas. Tissue expansion can also be helpful in obtaining tissue with a good match. ${ }^{16}$ "Splinting" can be achieved by suturing the lids together for one to two weeks. This is particularly helpful when after prolonged exposure the conjunctiva become markedly swollen and will not return to a normal position without encouragement. 


\section{Neck}

These contractures can be severe, with attachment of the mentum or lip to the sternum. In severe cases in children, mandibular growth can be affected with up to a one $\mathrm{cm}$ gap between upper and lower teeth after release. Severe cases may involve speech and oral hygiene problems, and the proximity of the mouth to the operative site is associated with an increased wound infection risk. Dental hygiene should be dealt with prior to surgery for the burn contracture. Intubation can be quite difficult, and this should be done by an experienced anaesthetist , preferably with fiberoptic introducing facilities. Often in adults it is best to release the contracture under local anaesthetic, and the skin can be harvested with a spinal anaesthetic or a general anaesthetic following release. Any grafting in the neck must be done on a dry bed. All unsightly scars should be excised to obtain good cosmetic results. Grafting can be done the following day if in spite of adrenaline and cautery there is continued oozing. Full thickness grafts from the clavicular area give excellent cosmetic results, while for larger areas the supra-pubic or groin areas can be used, where the horizontal scar will be hidden by the underwear. ${ }^{2,18}$ No skin for the face and neck should be meshed as it gives a very poor cosmetic result. Tie-over dressings with a large bulk should keep the graft in place, and after opening the dressing the patient should lie with a pillow behind the shoulders for up to six to twelve months. A neck splint is helpful in preventing recurrent contractures. Tissue expanders if available give good results. Free skin flaps from the groin or scapular area have also been reported with good cosmetic results. ${ }^{19}$

A cervical collar must be worn for six months to one year.

\section{Axilla}

Failure to use a flap in this area will lead to a high incidence of recurrence. For tight bands, a five-flap release (Fig.4) ${ }^{20}$, transposition flaps ${ }^{21}$ Z-plasties, or V-Y plasties are ideal. For broader contractures, medial arm or parascapular flaps are very good giving good functional results. ${ }^{21,22,23}$ The axial bilobed

\section{Fig. 4}

and thoracodorsal perforator-based flaps can also be used with good functional and cosmetic results. ${ }^{24,25}$ Rarely, in severe adduction with the arm attached to the chest wall the latissimus dorsi flap can be used. The flap must cover the joint line and defects on the chest and arm can be skin-grafted. Post-operative splinting is uncomfortable but necessary for a short time only when flaps are used.

\section{Elbow}

In adults this joint becomes very stiff and obtaining full release at the first attempt is unusual. After the first release, rigorous physiotherapy is needed, after which a second procedure should get full release. Again, a thin band is well handled by a fiveflap (Fig. 5) release or V-Y or Z-plasties. Larger contractures can be managed by a transposition fasciocutaneous flap to cover the joint, and remaining defects above or below the flap can be grafted. Alternatively a single or multiple bipedicle flap where one of the flaps lies over the joint can be used, with skin grafting to the defects above and below the joint. ${ }^{26,27}$

\section{Wrist}

This joint usually can be released and grafted without exposure of tendons or ligaments and with a low risk of recurrence if appropriate splinting is used. If needed a groin flap will provide good coverage.

\section{The Hand}

Joint stiffness is a significant problem in the hand, especially in the adult. Salisbury recommends capsulectomies of both metacarpal phalangeal and proximal interphalageal (PIP) joints if necessary to improve the range of motion, power and dexterity. ${ }^{28}$ The importance of coordinating proposed operations with the involved therapist cannot be overemphasized. Chronic pain is a common problem and interferes with successful therapy. Salisbury notes that "the thumb is $50 \%$ of the hand." Considerable efforts must be made to fully release the thumb, which in severe adduction contractures may involve carpometacarpal joint release and adductor release. A forearm flap may be used to give good skin coverage. ${ }^{29}$

\section{Metacarpal Phalangeal Joints}

Release of flexion contractures generally will have a high success rate, and again full thickness, or super-full thickness skin grafts, will give good results. ${ }^{910}$ It is much more difficult to obtain full release from extension contractures. The collateral ligaments become tight in the extended position, preventing full flexion of the joint even when the skin is fully released. Options in this situation are:

a) Obtain maximal release of the contracture and then use post-operative physiotherapy to gain further range of motion in flexion.

b) Release of the joint capsule and collateral ligaments at 
the first procedure. This may require flap coverage which would require either a groin or radial forearm flap, or raising a flap from the proximal dorsum of the hand which would then cover the MCP joints. ${ }^{30}$ The viability of this flap could be in question.

Whether dealing with flexion or extension release, K-wires should be used for two to four weeks post-operatively, as plaster alone is often unable to maintain an adequate position. After this period aggressive therapy is started with splinting between therapy sessions. By six weeks post-operatively night splinting alone should suffice.

\section{Interphalangeal joints}

Good results can be expected with full thickness skin grafts or split- thickness plantar grafts. Small grafts can be obtained from the ulnar aspect of the wrist crease.${ }^{31}$ The digital nerves and vessels are significantly at risk during release and must be carefully preserved. ${ }^{28}$ They often tent up somewhat after release but the graft will easily take over top of them if tieover dressing are used. A separate release incision for each joint is required. K-wire fixation is required for two to four weeks post-operatively. Marked ulnar deviation of the little finger or radial deviation of the thumb can be seen, and these likewise can be released with expectation of good results. As pointed out above, where possible the use of local flaps is preferred. Occasionally when tendons are exposed during release a cross finger flap may be of use. With severe contractures, at times a PIP arthrodesis will improve function and decrease pain. ${ }^{28}$

\section{Hip}

Z- or V-Y plasties may suffice here. If tensor fascia lata contracture is an impediment to release, it can be released as well and a lateral thigh transposition flap used to cover the area. $^{2,6}$ The TFL myocutaneous flap is very helpful in severe contractures., ${ }^{2,6,11}$

\section{Knee}

Great care must be taken not to injure the popliteal structures. A five skin flap release can be effective here. If a long web is present between thigh and calf, it may be possible to obtain near complete coverage with inter-connecting zig-zag incisions similar to a syndactaly release. Lateral or medial fasciocutaneous flaps or gastrocnemius flaps can be helpful in coverage and prevention of recurrence. ${ }^{6,11,12}$ A bipedicle flap to cover the polpiteal area with skin grafts above and below it has been reported, and in severe cases multiple bipedicle flaps can be used. ${ }^{26}$ Free or distally based anterolateral thigh flaps have also been described with good results. ${ }^{32}$

\section{Ankle}

These can be very severe, occasionally presenting with a "peg leg" type of deformity (Fig.6a\&b). The type of skin on the sole surface must be assessed prior to consideration of release. If the sole skin is also scarred and of poor quality there is unlikely to be much benefit from release as they will develop recurrent ulceration of the scarred skin on weight-bearing. In these cases it is better to give an orthosis to assist in wearing shoes. More often, the skin on the sole is healthy and one can expect good results. Division of tendons and vessels on the dorsum of the ankle may be necessary to obtain full release. This does not present a functional problem as usually in severe cases there are no toes remaining. Two procedures are often required for full release. Large $\mathrm{K}$-wire fixation is required postoperatively. When the joint is exposed the sural nerve flap is of great use or if there is good skin above the joint an inferiorly based medial or lateral flap can be of use.

\section{Toes}

Careful consideration and discussion with the patient/ family is required to determine what benefit will be obtained from release. Skin graft on the sole of the foot fares poorly, and functional gains are often minimal. However, in tropical climates it is important to have an adequate first web space

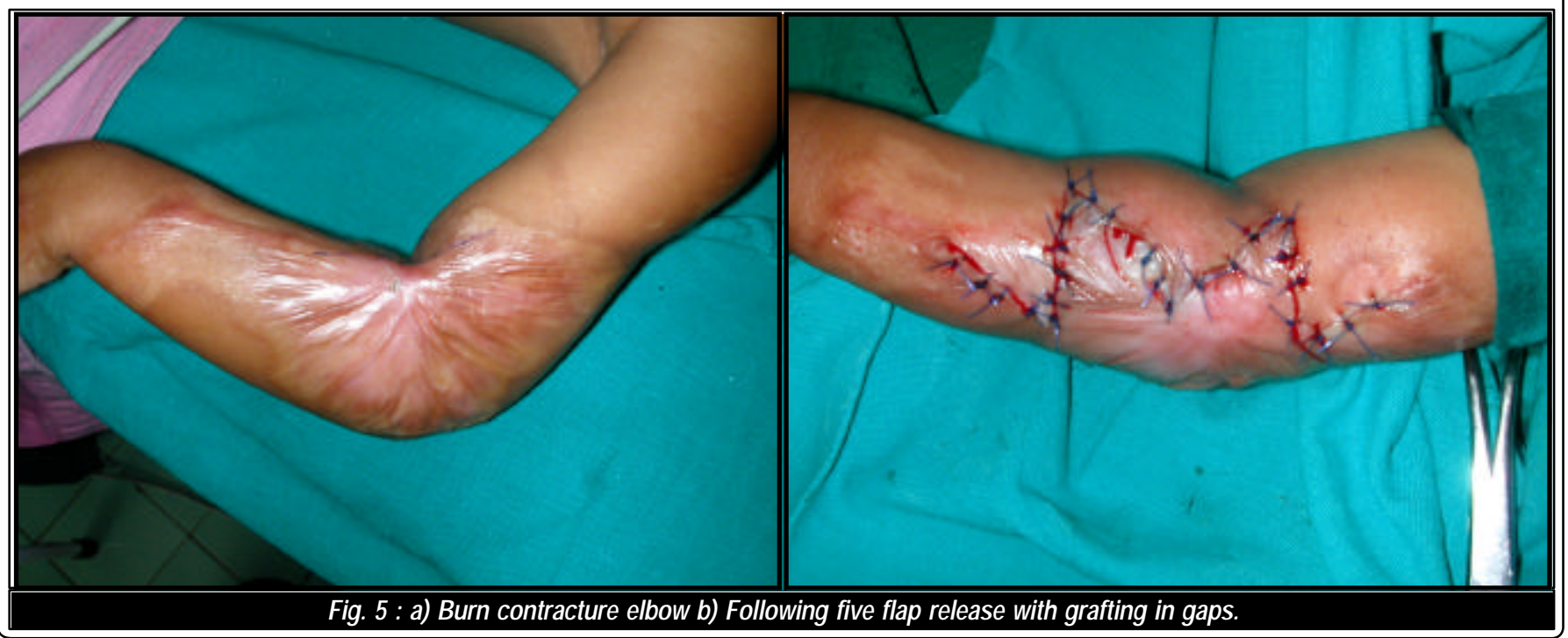




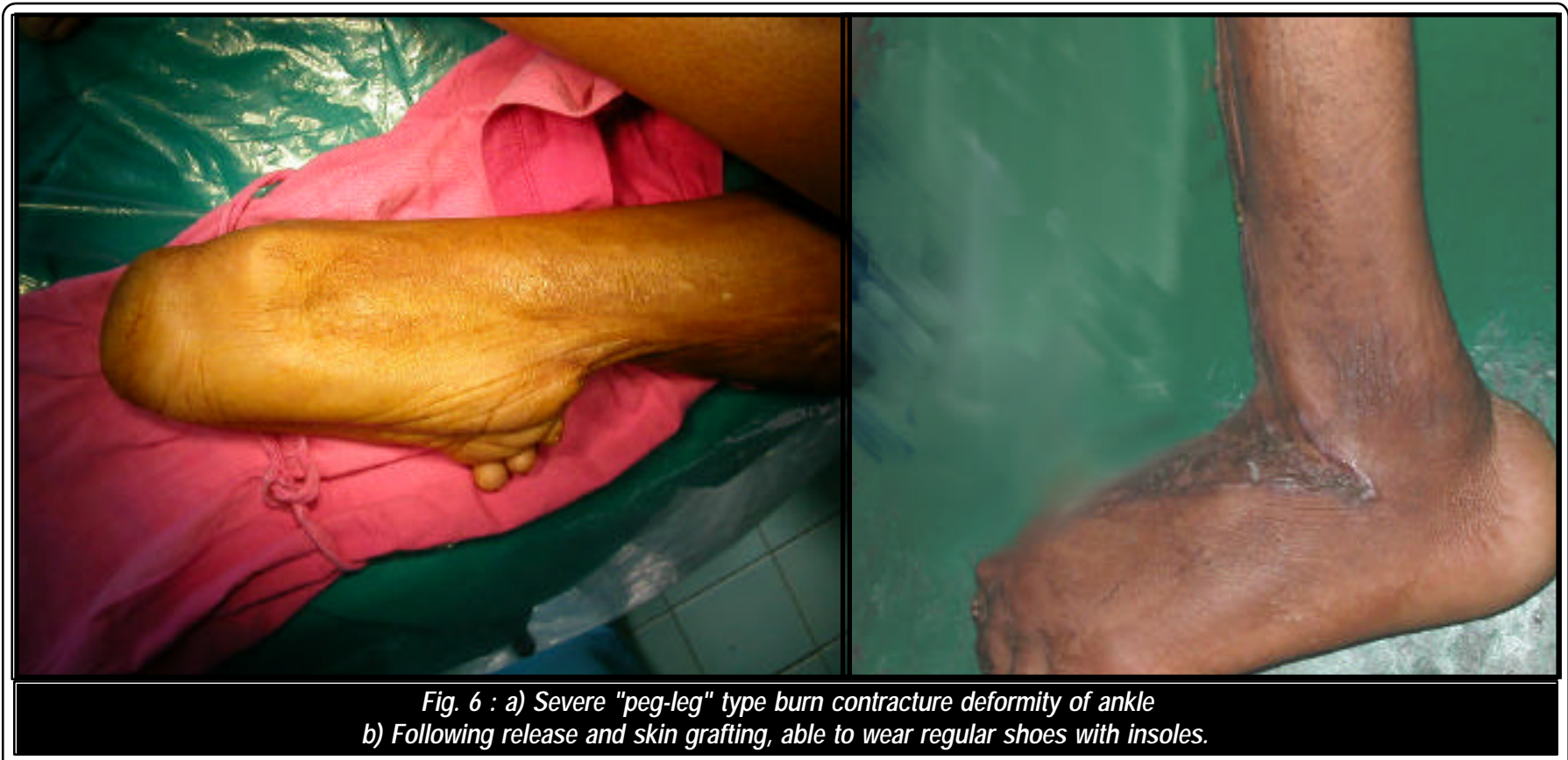

for wearing sandals, and severe contractures can make walking or wearing footwear difficult. The comments under interphalangeal joints above apply here as well.

\section{POST-OPERATIVE CARE}

Appropriate splinting is required. In the digits and ankle, Kwire fixation is needed, which is usually removed at four weeks to reduce the risk of pin-track infection. For other joints, plaster or other moldable material is ideal.

In clean cases, following skin grafting the first dressing is carried out at one to two weeks depending on the practice of the surgeon. One can expect near $100 \%$ take in $95 \%$ of grafts in clean cases. Areas of loss can be re-grafted after the area is clean.

The importance of post-operative therapy, especially in the burned hand, cannot be overemphasized. Regular therapy sessions by a cooperative patient are essential to obtain a maximal range of functional motion. Adequate pain control may be necessary during these sessions. Prolonged splinting is required following skin grafting procedures but therapy sessions should be started within two to three weeks following surgery, removing the splint for each session. By six weeks following the surgery night splinting alone should be sufficient, and may need to be continued for up to a year.

In the hand, an outrigger device allowing active flexion with passive extension is valuable but difficult to provide in the developing country situation. Night splinting is continued for at least six months following grafting. If flaps were used prolonged splinting is usually not required. If a second procedure is required one should wait at least six to twelve months between procedures.

\section{REFERENCES}

1. Pandya AN. Principles of treatment of burn contractures. Repair Reconstr 2002; 2 (2) :12-13.

2 Salisbury RE, Bevin AG. Atlas of Reconstructive Bum Surgery. Philadelphia: W.B.Saunders Company 1981.

3. Dias AD. Postburn contractures. Plast Reconstr Surg 2001; 108 (2) $575-6$.

4. Yildiram S, Avci G, Akan M, Misirlioglu A and Akoz T. Anteriolateral flap in the treatment of postbum contracture of the knee. Plast Reconstr Surg 2003; 111 (5) , 1630-1637.

5. Peacock E, Beasley RW. Of burned hand and upper extremity. In: J.G.MoCarthy (ed) : Plastic Surgery Volume 8; p5452-5482. Philadelphia: W.B. Saunders.Co 1990.

6. Ramakrishnan M, Hanumadass MD. Handbook of Burns management. Delhi: Jaypee Bros Medical Publishers 1991.

7. Bunyan AR, Mathur BC. Medium thickness plantar skin graft for management of digital contractures. Burns 2000; 26 (6) 575-80.

8. Tanabe HY, Aoyagi A, Tai Y et al. Reconstruction for plantar skin defects of the digits using plantar demal grafting. Plast Reconstr Surg 1998; 101:992-995.

9. Iwagwu $\mathrm{FC}$, Wilson $\mathrm{D}$, Bailie $\mathrm{F}$. The use of skin grafts in $\mathrm{PBC}$ release. Plast Reconstr Surg 1999; 103 (4) : 1198-204.

10. Pensler JM, Steward R, Lewis SR, Herndon DN. Reconstruction of the burned palm: Full-thickness versus split-thickness skin grafts- long-term follow-up. Plast Reconstr Surg 1988; 81:4651. 
11. Masquelet, A. Gilbert, A. Flaps in Limb Reconstruction 1995 Lippincott Co

12. Strauch B, Vasconez LO, Hall-Findlay EJ (eds). Grabb's Encylopedia of Flaps. Toronto: Little, Brown and Co, 1990.

13. Goodacre T. Surgical techniques: the V to Y plasty. Repair Reconstr 2001; 2 (2) : 13.

14. Ebbehoj J. Y to V instead of $\mathrm{Z}$ to N. Burns Incl Therm Inj 1983; $10: 121-125$.

15. Ullmann Y Lerner A. A New approach to deal with post burn knee contractures. Burns 2003; 29 (3) 284-6.

16. Kawashima $\mathrm{T}$, Yamada $\mathrm{A}$, Ueda $\mathrm{K}$ et al. Tissue expansion in facial reconstruction. Plast Reconstr Surg 1993; 94:944-951.

17. Achauer BM, Adair SR. Acute and reconstructive management of burned eyelid. Clin Plast Surg 200027 (1) 87-9.

18. Cronin ID. Bum scar contracture of the neck. In: Stark RB (ed) Plastic Surgery of the Head and Neck; p1433. New York: Churchill Livingstone 1987.

19. Ohkubo E, Ohmori K. Restoration of the anterior neck surface in the burned patient by free groin flap. Plast Reconstr Surg $1991 ; 87: 276$.

20. Hirshowitz B, Karev A. Axillary Reconstruction: Five-skin flap. In Strauch B, . Vasconez, Luis, Findlay, EH (eds); Grabbs's Encyclopedia of Flaps; p 1228-1229. Toronto: Little Brown and Company1990.

21. Armstrong DP. Axillary Reconstruction: Transposition and advancement skin flaps. In: Strauch B, . Vasconez L, Findlay EH (eds); Grabbs 's Encyclopedia of Flaps, p1221-1227. Toronto: Little Brown and Company 1990.
22. Tanaka A Hatko M. An evaluation of functional following surgical corrections of severe bum scar contracture in the axilla. Bums 2003; 29 (2) 153-7.

23. Tiwari P, Kalra GS, Bhatnagar SK Fasciocutaneous flaps for burn contractures of the axilla. Burns 1990, 16, 150-2.

24. Karacalar A, Guner $\mathrm{H}$. The axial bildbed flap for $\mathrm{BC}$ of the elbow. Burns 2000; 26 (7) 628-33.

25. Kim DY, Cho SY, Kim KS. Correction of axillary burn scar contracture with the thoracodorsal flap. Ann Plast Surg 2000; $44(2): 181-7$

26. Prakash V. A new concept for the management of postburn contractures. Plast Reconstr Surg 2000;106 (1) 233-4.

27. Prakash V, Baja SD. A new concept for the treatment of postburn contracture of the elbow. Ann Plast Surg 2000; 45 (3) 339.

28. Salisbury RE. Reconstruction of the burned hand. Clin Plast Surg 2000;27:65-69.

29. Costa,H, Soutar, D.S. The distally based island posterior interosseous flap. Br J Plast Surg 1988; 41:221-226.

30. Fritschi E. Reconstructive Surgery for Leprosy. The Leprosy Mission, New Delhi, 1984.

31. Park S, Hata Y, Ito O et al. Full-thickness skin graft from the ulnar aspect of the wrist to cover defects on the hand and digits. Ann Plast Surg 1999; 42: 129-131.

32. Yildiram S, Avci G, Akan Met al. Anterolateral thigh flap in the treatment of postburn flexion contractures of the knee. Plast Reconstr Surg 2003;111:1630-1637.

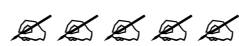

